\title{
Défaut de fourchaison chez le Pin laricio de Corse (Pinus nigra Arn. ssp. laricio var. corsicana Loud) : décomposition du phénomène en vue de l'amélioration génétique
}

\author{
Catherine PORTEFAIX \\ avec la collaboration technique de Marc FAUCHER et Dominique VEISSE \\ INRA, Station d'Amélioration des Arbres forestiers \\ Centre de Recherches d'Orléans \\ Ardon, F 45160 Olivet
}

\begin{abstract}
Résumé
Une décomposition du défaut de fouchaison chez le Pin laricio de Corse a été réalisée sur les verticilles 1983 et 1984 d'un test de descendances maternelles âgé de 9 ans, situé en forèt d'Orléans.

La présence de pousses secondaires ou plus précisément d'allongements secondaires sur les branches latérales, bien que sensible à l'effet année, semble être le principal facteur explicatif de rapparition des 2 types de défauts: fourches et ramicornes.

L'utilisation complémentaire d'une note d'angle moyen de branchaison, plus stable dans le temps, permet d'évaluer l'importance des causes de la fourchaison autres que le polycyclisme.

Dans la population d’amélioration étudiće, ces 2 caractères s'avèrent être de bons critères prédicteurs de la fourchaison, avec une variabilité génétique intéressante et une héritabilité individuelle assez élevée $(0,3-0,4)$. L'absence de corrélation génétique significative entre hauteur totale et angle de branchaison réduit l'importance de l'antagonisme vigueur-niveau de fourchaison à la seule corrélation génétique positive entre hauteur totale et polycyclisme.

Le meilleur compromis au niveau de l'espérance des gains génétiques scra obtenu grâce à la construction d'un indice de sélection combinant les 3 caractères: hauteur totale, polycyclisme et angle de branchaison.
\end{abstract}

Mots clés: Pinus nigra ssp. laricio var. corsicana, fourchaison, polycyclisme, angle de branche, héritabilité. variance génétique, corrélation génétique, gain génétique.

\section{Introduction}

Si le Pin laricio de Corse allie une excellente forme du fût à une finesse de branches, de graves défauts de fourchaison peuvent néanmoins affecter la qualité de la tige produite. L'absence de fourchaison est donc, au même titre que la vigueur, un des critères de sélection utilisés dans le programme d'amélioration de l'espèce.

La présence de branches faisant avec laxe du fût un angle inférieur à $30^{\circ}$ étant jugée défavorable, le défaut est qualifié conventionnellement de ramicorne lorsque le 
diamètre à la base de la branche est inférieur à la moitié de la tige principale et de fourche lorsqu'il est supérieur (fig. 3).

Le système de notation utilisé jusqu'à présent pour apprécier l'importance des défauts n’apparaît pas très satisfaisant. Les faibles valeurs de variance génétique et d'héritabilité indivuelle font de la note de fourchaison un critère de sélection peu efficace car trop influencé par les conditions de milieu, conditions climatiques de l’année, mais aussi conditions édaphiques (tabl. 1). L'espérance de gain génétique attendu par sélection au taux de 10 p. 100 n'atteint pas 3 p. 100.

TABILAU I

Corrélations génétiques entre la fourchaison, le polycyclisme et la hauteur totale dans les 2 tranches du verger à graines de Pin laricio de Corse situé dans le Lot.

Genetic correlations, between forking. polycyclism and total height in 2 sections of the Corsican Pine seed orchards located in Lot.

\begin{tabular}{|c|c|}
\hline \multicolumn{2}{|c|}{ Note de fourchaison cumuléc sur 1983 et 1984} \\
\hline 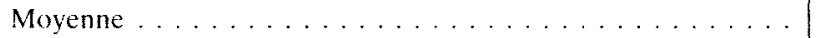 & 93,72 \\
\hline Ecart-type $\ldots \ldots \ldots \ldots \ldots \ldots \ldots \ldots$ & 7,77 \\
\hline Variance génétique additive $\sigma_{A}^{2} \ldots \ldots \ldots \ldots$ & 10.4 \\
\hline 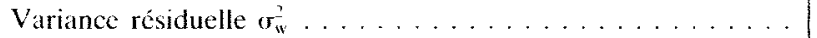 & 61,05 \\
\hline Héritabilité individuelle $\mathbf{h}^{2} \ldots \ldots \ldots \ldots \ldots$ & 0,16 \\
\hline \multicolumn{2}{|l|}{$\begin{array}{l}\text { La note de fourchaison (FOUR) se définit de la façon suivante: } \\
\text { FOUR }=100-5 \times(\mathrm{R})-10 \times \text { (F) } \\
\text { ou } R \text { est le nombre de ramicomes observes sur les } 2 \text { verticilles } 1983 \text { et } 1984 \text {, } \\
\text { F est le nombre de fourches observécs sur les } 2 \text { verticilles } 1983 \text { et } 1984 \text {. }\end{array}$} \\
\hline
\end{tabular}

Cette étude se propose de décomposer le syndrome de fourchaison dans le double objectif suivant :

- au niveau descriptif, pour une meilleure appréciation de l'importanee des facteurs explicatifs de la fourchaison, connus en forêt d'orléans ;

- au niveau méthodologique pour l'identification d'un ou plusieurs critères prédicteurs optimaux pour la sélection, sous déterminisme génétique plus étroit.

Les problèmes liés à la structure génétique de la population étudiée sont volontairement laissés de côté ici pour être développés ultérieurement dans une thèse de troisième cycle.

\section{Matériel et méthodes}

\subsection{Matériel végétal}

L'ensemble des observations porte sur un test de descendances maternelles âgé de 9 ans depuis la graine et situé en forèt domaniale d'Orléans sur un sol siliceux profond. 
Les 48 familles étudiées dans ce test appartiennent à 4 peuplements de Sologne. Elles constituent une partie de la population d'amélioration d'origine Centre et sont représentées dans les vergers à graines du Lot. Chaque descendance est représentée en moyenne par 49 individus, répartis dans des blocs incomplets aléatoires par parcelle unitaire de 4 arbres.

Une étude réalisée par Rabat (1985) sur Pins laricio de Corse et de Calabre a montré que le phénomène de fourchaison s'exprime très tôt dans la vie de l'arbre. Plus de 70 p. 100 des arbres défectueux âgés de plus de 40 ans présentaient un défaut dès l'âge de 15 ans.

A l'issue d'une étude préliminaire, un échantillon de tous les arbres défectueux appartenant à 15 familles (soit 443 au total), a été constitué. Une analyse discriminante sur les 3 caractères, note de fourchaison (FOUR), hauteur totale en 1984 (HT84), et nombre de pousses secondaires a testé la bonne représentativité de cet échantillon sur lequel s'est limitée l'étude descriptive de la fourchaison.

\subsection{Recherche de descripteurs de la fourchaison sur l'échantillon}

\subsection{Choix des 2 années d'étude}

Un minimum de 2 années d'étude est nécessaire pour apprécier l'influence des facteurs environnementaux sur l'importance et la nature du phénomène de fourchaison, qui en ne touchant qu'une partie de l'arbre, affecte la valeur marchande de la tige entière. Les observations ont été réalisées à la fin de la saison de végétation 1985. Mais, pour tenir compte de l'ouverture de l'angle moyen d'insertion des branches l'année suivant la formation du verticille, seules les pousses 1983 et 1984 ont été étudiées. Il faut dès à présent noter l'absence de corrélation individuelle existant entre les 2 notes FOUR 83 et FOUR $84(\hat{\mathbf{r}}=0,004 \mathrm{~ns})$.

\subsection{Facteurs explicatifs de la fourchaison}

\subsection{Dégâts occasionnés par les insectes et les champignons}

La mineuse des bourgeons (Rhyacionia buoliana), ennemi principal du Pin laricio de Corse dans le jeune âge, semble n'avoir affecté que faiblement l'ensemble des dispositifs de la forêt d'Orléans. Toutefois, les attaques observées sur les individus de l'échantillon ont été comptabilisées afin d'estimer l'importance et la nature des dégâts occasionnés par l'insecte, ceci pour les 2 années 1983 et 1984.

Après un premier passage sur la plantation, il s'est avéré intéressant de prendre en compte les attaques de rouille courbeuse (Melampsora pinitorqua) en 1983, mais surtout en 1984, où elles ont été importantes sur pousses latérales.

\subsection{Influence du polycyclisme}

Un individu polycyclique a des pousses annuelles constituées de deux ou plusieurs cycles morphogénétiques: le premier cycle est appelé pousse primaire, le ou les suivants pousse secondaire.

Chez de nombreuses espèces, le polycyclisme semble avoir une influence directe néfaste sur la fourchaison, Carvel. (1956) sur Pinus resinosa, Rudolph (1964) sur Pinus banksiana, WEST \& LEDIG (1964) sur Pinus sylvestris ont mis en évidence la multiplication des fourches lors de la formation de pousses d'Août. 
Chez le Pin maritime, il existe des corrélations hautement significatives au niveau génétique $(0,71-0,76)$ entre absence de pousse secondaire et absence de fourche (CRISAN, 1977).

Dans les 2 premières tranches du verger à graines de Pin laricio de Corse, il a déjà été trouvé une corrélation génétique hautement significative entre polycyclisme et note de fourchaison $(-0,69,-0,75)$. La corrélation génétique entre hauteur totale et polycyclisme était significative dans un cas $(0.400)$, non significative dans l'autre. Il faut souligner qu'à la différence du Pin maritime landais, les pousses secondaires, réalisées sur la tige principale sont souvent trop courtes pour participer de façon notable à l'élongation totale de l'arbre.

Afin de préciser l'influence du polycyclisme sur l'apparition de défauts de fourchaison, les notations suivantes ont été réalisées sur l'échantillon de 443 arbres:

- le nombre total de pousses secondaires sur rameaux latéraux subterminaux pour chacun des verticilles 1983 et 1984 (PA83, PA84);

- le nombre total de pousses secondaires sur rameaux latéraux subterminaux ayant occasionné l'apparition de défauts, fourche ou ramicorne, pour chacun des verticilles 1983 et 1984 (PAD 83, PAD 84).

\subsection{Angle de branchaison ou branchaison ou type d'insertion}

L'étude réalisée par Arbez \& Millier (1972) sur Pin laricio de Calabre a souligné l'opportunité d'une mesure d'angle de branchaison. Si la variabilité du caractère est relativement faible dans le test étudié, certaines descendances apparaissent particulièrement fastigiées. De plus, l'héritabilité individuelle calculée est relativement élevée $(0,38-$ $0,54)$.

Chez le Pin sylvestre (Ehrenger, 1963), l'angle de branchaison apprécié par une valeur brute comprise entre 0 et $90^{\circ}$ est également héritable $(0,4-0,8$ selon les verticilles étudiés).

Il nous est apparu intéressant d'intégrer dans cette étude une mesure exploratoire d'angle de branchaison, non seulement afin d'apprécier son importance en tant que composante de la fourchaison, mais aussi afin d'obtenir une première estimation de ses paramètres génétiques (variance génétique, héritabilité, corrélations génétiques) chez le Pin laricio de Corse.

Compte tenu de la difficulté qui existe à mesurer une valeur brute d'angle (choix de la ou des branches à mesurer, précision et rapidité de la mesure), une note globale appréciant un angle moyen par verticille lui a été préférée. Testée sur Douglas (JARRET, 1978) une telle notation en classe s'est avérét satisfaisante comparée aux mesures d'angle réel. La corrélation pour la valeur moyenne des arbres entre les 2 types de mesure était de $-0,83$.

Sur l'ensemble des 48 descendances, une notation en 4 classes de 1 à 4 a été utilisée pour chacun des 2 verticilles 1983 et 1984 :

- note 1: toutes les branches sont quasi horizontales (fig. 1a);

- note 2 : quelques branches sont à faible angle d'insertion (fig. 1b) ;

- note 3 : la majorité des branches sont à angle d'insertion moyen à faible (fig. 1c) ;

- note 4 : toutes les branches sont à angle d'insertion faible (fig. 1d). 


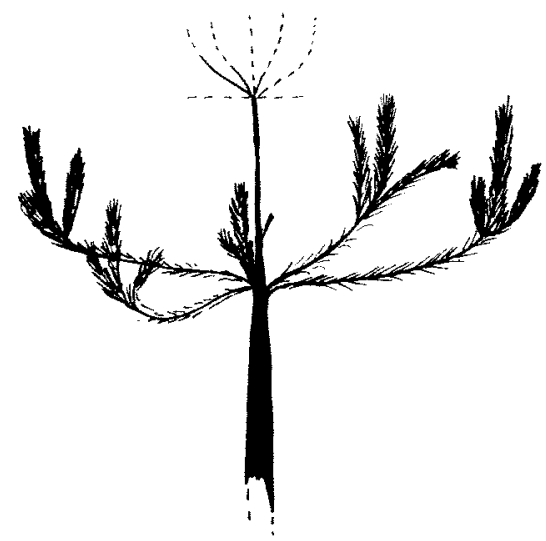

(1a)

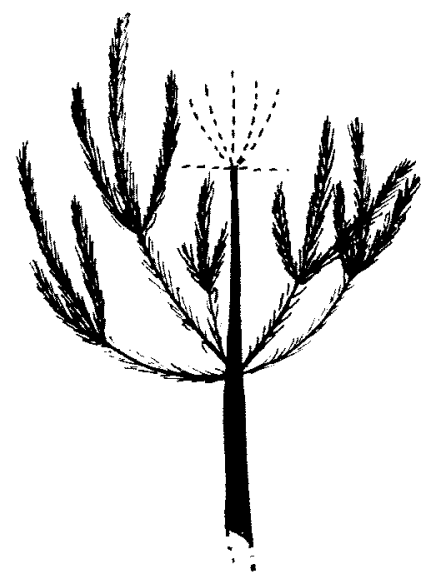

$(1 c)$

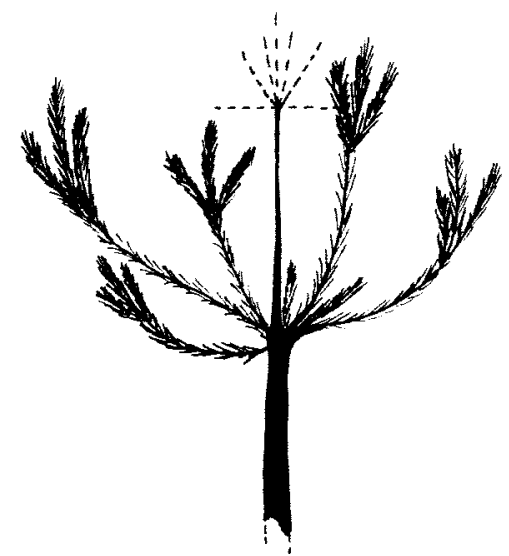

(1b)

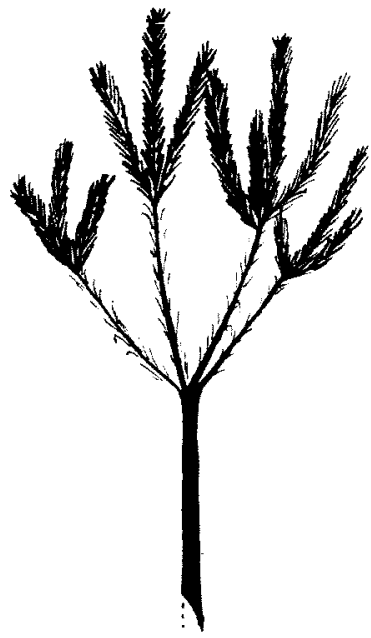

(1d)

Fig.

Echelle de notation utilisée pour l'appréciation de l'angle moyen de branchaison. Notation scale used to measure average branch angle.

a. $\operatorname{note}=1 ;$ b. note $=2 ;$ c. note $=3 ;$ d. note $=4$.

Cette notation rend compte d'un type d'angle moyen d'insertion des branches au niveau d'un verticille donné, en intégrant fourches et ramicornes. 


\subsection{Dégâts accidentels}

Les défauts à origine accidentelle certaine (bris, avortement accidentel du bourgeon terminal) ont été répertoriés.

Tous les autres défauts ont été considérés comme ayant une "origine non déterminée $»$.

\subsection{Méthodes de calcul des paramètres génétiques}

Après ajustement préalable des données au facteur bloc, le calcul de paramètres génétiques a été réalisé sur l'ensemble des 48 descendances. Le test de Kullback n'a pas conduit à rejeter l'hypothèse d'homogénéité des matrices de variances-covariances entre familles calculées pour les 4 peuplements étudiés. C'est pourquoi le modèle d'analyse de variance multivariable est un modèle hiérarchique à 2 facteurs: peuplement et famille :

$$
Y_{i j k}=\mu+P_{i}+F_{j(i)}+\varepsilon_{i j k}
$$

$\mu$ représente la moyenne générale du caractère $Y$;

$P_{i}$ et $F_{j(i)}$ sont respectivement les effets moyens des facteurs peuplement et famille dans peuplement ;

$\varepsilon_{i j k}$ est la résiduelle.

Le facteur peuplement étant considéré comme fixe, seule la matrice de variancecovariance entre familles $\mathrm{Z}$ f sera estimée à partir de l'espérance des carrés et coproduits moyens (tabl. 2).

\section{TABIFAU 2}

Tableau d'analyse de variance utilisée pour l'estimation des paramètres génétiques. Analysis of variance used for the estimation of the genetic parameters.

\begin{tabular}{l|r|c|c}
\hline \hline Source de variation & d.d.l. & $\begin{array}{c}\text { Somme des carrés } \\
\text { des écarts moyens }\end{array}$ & $\begin{array}{c}\text { Espérance } \\
\text { des carrés moyens }\end{array}$ \\
\hline Peuplement ...... & 3 & $\mathrm{CM}_{\mathrm{p}}$ & $\sigma_{w}^{2}+\mathrm{n}_{\mathrm{p}} \Phi_{\mathrm{p}}^{2}$ \\
Famille/peuplement . & 44 & $\mathrm{CM}_{\mathrm{P} / \mathrm{p}}$ & $\sigma_{\mathrm{w}}^{2}+\mathrm{n}_{1} \sigma_{\mathrm{l}}^{2}$ \\
Résiduelle ...... & 2337 & $\mathrm{CM}_{\mathrm{w}}$ & $\sigma_{\mathrm{w}}^{2}$ \\
\hline
\end{tabular}

$\sigma_{w}^{2}$ et $\sigma_{i}^{3}$ sont les variances résiduelle ef intertamille

$\mathrm{n}_{1}$ est le nombre moycn d'individus par famille

$$
n f=\left(n . .-\sum_{i=1}^{p} \sum_{j}^{q i} n_{i}^{2} / n_{i}\right) /(q-p)
$$

n.. cst le nombre total d'individus.

$\mathrm{n}_{\mathrm{jj}}$ est le nombre d'individus de la famille $\mathrm{j}$ issu du peuplement $\mathrm{i}$.

$n_{i}$ est le nombre d'individus du peuplement $i$.

$\mathrm{p}$ est le nombre de peuplements.

$q$ cst le nombre total de familles.

$\mathrm{q}_{\mathrm{f}}$ est le nombre de familles représentant le peuplement $i$.

$$
\Phi_{\mathrm{p}}^{2}=\frac{1}{(\mathrm{p}-1)} \stackrel{\mathrm{i}}{\stackrel{\mathrm{L}}{=}}, \mathrm{n}_{\mathrm{i}}, \mathrm{p}_{\mathrm{i}}^{2}
$$

De même un modèle multivariable, les covariances inter et intrafamilles sont estimées à partir de l'espérance des coproduits moyens. 
En supposant que les descendances maternelles obtenues par pollinisation libre sont composées exclusivement de demi-frères, que la consanguinité moyenne dans les peuplements d"origine est nulle et que les arbres-mères ne sont pas apparentés, la variance entre famille $\sigma_{f}^{\frac{?}{e}}$ estime $1 / 4$ de la variance génétique additive soit :

$$
\sigma_{\mathrm{A}}^{2}=4 \sigma_{\mathrm{i}}^{2}
$$

De même la covariance génétique additive entre 2 caractères $X$ et $Y$ s’écrira:

$$
\operatorname{COV}_{\mathrm{A}}(\mathrm{X}, \mathrm{Y})=4 \operatorname{COV}_{1}(\mathrm{X}, \mathrm{Y})
$$

Pourront donc être calculés :

- l'héritabilité individuelle $\mathrm{h}^{2}$ de chaque caractère, définie comme le rapport de la variance génétique additive à la variance phénotypique totale $\sigma_{p}^{2}$ (FAIconer. 1974).

$$
h^{2}=\frac{4 \sigma_{1}^{2}}{\sigma_{f}^{2}+\sigma_{w}^{2}}
$$

$$
\sigma_{p}^{2}=\sigma_{w}^{2}+\sigma_{i}^{2}
$$

-- le coefficient de corrélation génćtique $r_{A}$, entre les 2 caractères $X$ et $Y$ :

$$
r_{A}=\frac{\operatorname{Cov}_{\mathrm{i}}(\mathrm{X}, \mathrm{Y})}{\sqrt{\sigma_{\mathrm{i}}^{2}} \overline{(\mathrm{X}) \sigma_{\mathrm{i}}^{2}} \overline{(\mathrm{Y})}}
$$

- le coefficient de corrélation intrafamille $r_{w}$, entre valeurs individuelles de 2 caractères dans une même famille présentant une part génétique et une part environnementale non différenciables:

$$
r_{w}=\frac{\operatorname{Cov}_{w}(X, Y)}{\sqrt{\sigma_{w}^{2}(X) \sigma_{w}^{2}(Y)}}
$$

\section{Résultats et discussion}

\subsection{Description des facteurs de la fourchaison observés au niveau individuel sur l'échantillon}

Les moyennes des principaux descripteurs de la fourchaison ont été regroupées dans le tableau 3. L'année 1984 semble avoir été particulièrement favorable à l'expression de la fourchaison sur ce dispositif.

3.11. Importance des attaques de mineuse (Rhyacionia buoliana) et de rouille courbeuse (Melampsora pinitorqua)

Une seule attaque de mineuse des bourgeons a été dénombrée pour les 2 annéc. 1983 et 1984. Les 16 attaques de rouille, toutes observées en 1984 n'ont occasionné qu'à peine 6 p. 100 des défauts de l'année. Aussi, ni les attaques de mineuse, ni lés attaques de rouille ne peuvent être retenues comme facteurs explicatifs de la fourchaison observée en 1983 et 1984 sur ce dispositif de la forêt d'Orléans.

\subsection{Polycyclisme}

L'expression du polycyclisme semble être très liée aux conditions environnementales et en particulier aux conditions climatiques de l'année (tabl. 3). 


\section{TABleau 3}

Movennes at écart-rypes des caractires observés sur l'échantillon de 443 individus fourchus. Means and standard deviations of the traits observed on the 443 forked plants.

\begin{tabular}{|c|c|c|c|c|}
\hline \multirow[b]{2}{*}{ Caractères } & \multicolumn{2}{|c|}{1983} & \multicolumn{2}{|c|}{1984} \\
\hline & $\overline{\mathrm{x}}$ & $\sigma$ & $\overline{\mathrm{x}}$ & $\sigma$ \\
\hline Note de fourchaison & 98.95 & 2,98 & 96,58 & 5.96 \\
\hline Nombre de pousses secondaires & 1.21 & 1,474 & 0,97 & 1,283 \\
\hline $\begin{array}{l}\text { Nombre de pousses secondaires } \\
\text { ayant donné des délauts ..... }\end{array}$ & 0.15 & 0,391 & 0,36 & 0.639 \\
\hline Nombre de fourches $\ldots \ldots \ldots$ & 0.011 & 0.106 & 0,123 & 0.400 \\
\hline Nombre de ramicornes & 0.187 & 0.417 & 0.436 & 0.631 \\
\hline Angle de branchaison & 2.64 & 0.609 & 3.22 & 0.664 \\
\hline
\end{tabular}

\subsection{Effet année sur lexpression du polycyclisme}

C'est en 1983 que les conditions climatiques semblent avoir été les plus favorables à la formation de pousses secondaires: 48,5 p. 100 des arbres de l'échantillon étaient polycycliques en 1983 contre 39.7 p. 100 en 1984. Par contre, les nombres moyens de pousses secondaires par individu polycyclique sont très voisins : 2,44 en 1983 et 2,37 en 1984.

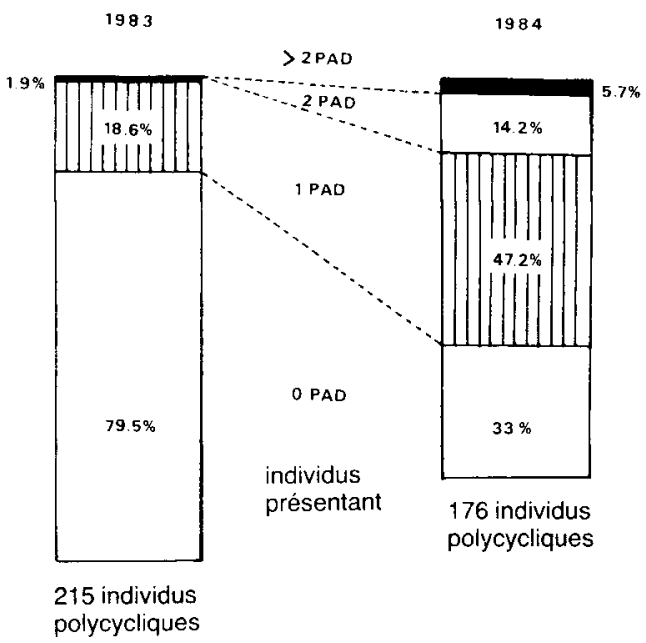

Fig. 2

Arbres présentant des pousses secondaires à l'origine de défauts (PAD).

Trees with lammas shoots inducing forking defects (PAD). 
II existe également un effet année sur le type de pousses secondaires formées (tabl. 3 et fig. 2) : en 1984, 67 p. 100 des arbres polycycliques ont vu, au moins une de leurs pousses secondaires être à l'origine d'un défaut contre seulement 20,5 p. 100 en 1983. Plus de 37 p. 100 des pousses secondaires sont à l'origine de l'apparition de fourches ou de ramicornes en 1984 , contre seulement 12,4 p. 100 en 1983. La corrélation individuelle entre les 2 caractères PAD 83 et PAD 84 est quasi nulle $(r=0,022)$.

L'observation des pousses secondaires formées en 1984 permet de préciser qu'il s'agit surtout d'allongements de bourgeons latéraux inférieurs à $10 \mathrm{~cm}$ plutôt que de véritables pousses d'août (fig. 3).

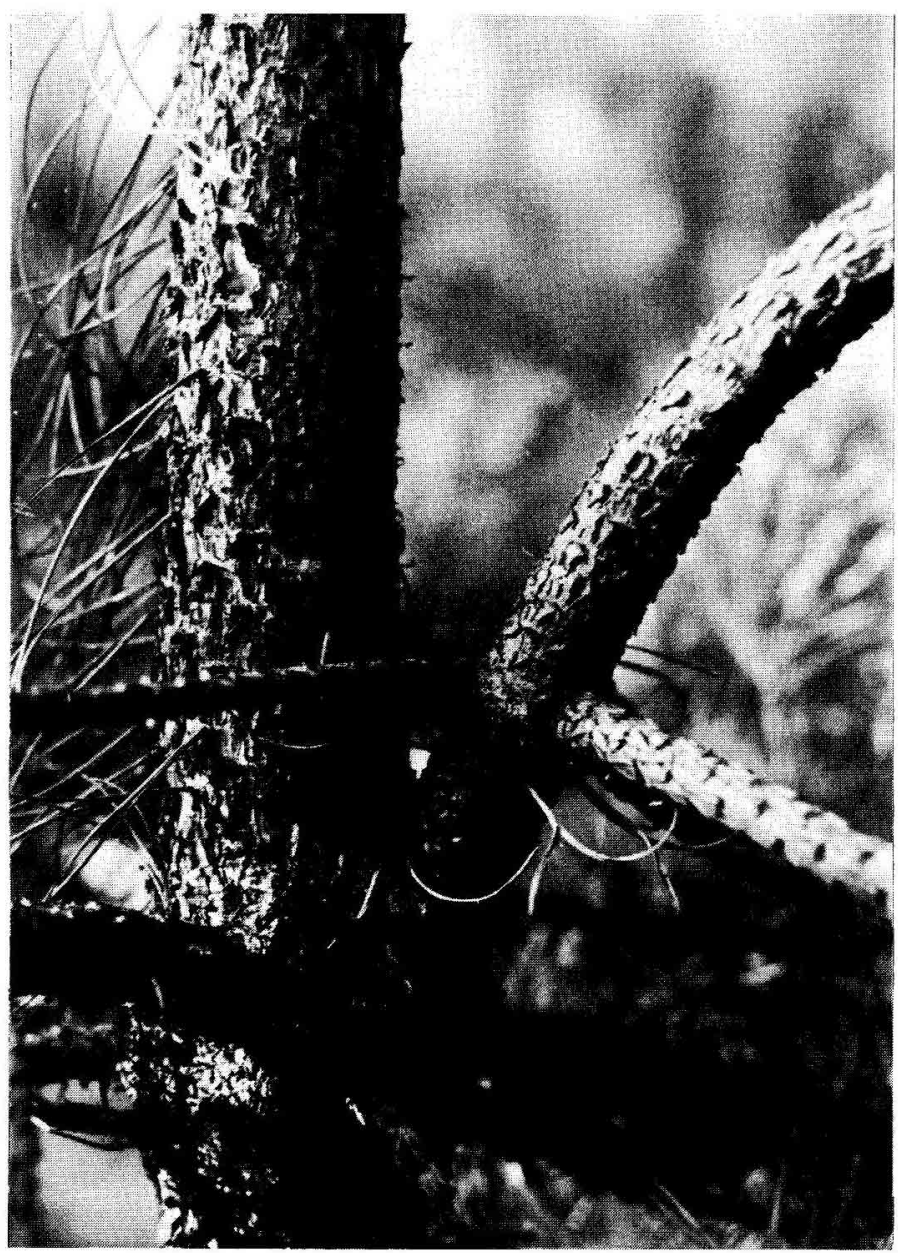

FIG. 3

Ramicorne occasionnée par l'allongement secondaire d'un bourgeon latéral. Ramicorne caused by a secondary elongation of a lateral bud. 


\subsection{Polycyclisme et nature des défauts observés}

En 1983, 74 p. 100 des arbres défectueux ont eu des défauts occasionnés par la présence de pousses secondaires contre $60 \mathrm{p}$. 100 en 1984. Le polycyclisme apparait être une composante essentielle de la fourchaison dès que les conditions de milieu sont favorables à son expression.

Le facteur polycyclisme explique à lui seul lapparition de 68 p. 100 des ramicornes, ceci pour les 2 années 1983 et 1984 (fig. 4). Si la cause principale de formation des fourches est bien la présence de pousses secondaires sur branches latérales, les attaques de rouille sont à l'origine de 13 des 55 fourches observées en 1984. Rappelons que ces fourches ne représentent cependant que $1 / 5$ des défauts observés.

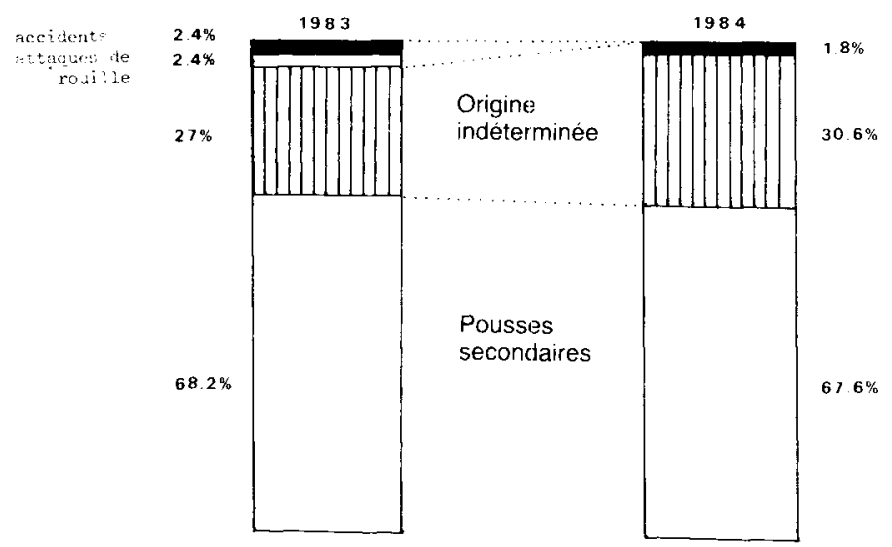

Fig. 4

Origine des ramicornes observées en 1983 et en 1984.

Origin of the ramicornes observed in 1983 and in 1984.

L'origine de près de 30 p. 100 des défauts observés, fourches ou ramicornes, a pu être déterminée.

\subsection{Angle de branchaison}

\subsection{Effet année}

L'évolution de la note de fourchaison entre 1983 et 1984 est parallèle à celle de l'angle de branchaison et correspond à une augmentation du nombre de défauts (tabl. 3).

Les corrélations individuelles entre les 2 valeurs d'angle A83 et A84 ont été calculées sur l'échantillon et sur l'ensemble du dispositif :

- sur échantillon (443 arbres) : $r_{\text {A } 3 \text {-A A 4 }}=0,367^{* *}$ significatif à 1 p. 100 ;

- sur l'ensemble des 48 descendances (3285 arbres) : $\mathrm{r}_{\mathrm{A} 83-\mathrm{A} 84}=0,442^{* *}$ significatif à 1 p. 100. 
Ces corrélations, bien que de valeur moyenne, montrent que l'angle de branchaison est beaucoup moins sensible à l'effet année que la note de fourchaison ou même le polycyclisme. Il faut noter également que les 2 verticilles étudiés sont susceptibles de voir leur angle moyen s'ouvrir au cours des prochaines années, le verticille 1984 étant décalé d'un an.

\subsection{Angle de branchaison et défauts de fourchaison}

La mesure d'angle de branchaison réalisée à l'aide d'une note, permet surtout une bonne discrimination des individus très défectueux (fig. 5). En effet, les arbres présentant une note égale à 4 ont eu en moyenne à la fois le plus grand nombre de fourches et de ramicornes.

Parmi les arbres non polycycliques, plus de $80 \mathrm{p}$. 100 des individus présentant au moins un défaut ont une branchaison fastigiée (note d'angle égale à 3 ou 4).

\subsection{Discussion}

Les deux types de défauts, fourche et ramicorne ne s'expriment pas dans les mêmes conditions de milieu mais ont pour cause essentiellement 2 phénomènes :

- le plus important dans ce dispositif en 1983 et 1984 semble avoir été le polycyclisme. En 1984, les conditions environnementales ont été à peine favorables à son expression, il s'est alors traduit par la présence d'allongements secondaires des bourgeons latéraux ; ces derniers conduisent le plus souvent à l'apparition de ramicornes l'année suivante.

En 1983, année plus favorable, il s'est formé de véritables pousses secondaires plus rarement à l'origine de défaut.

- une deuxième composante beaucoup plus stable avec les années semble être liée à la présence d'un faible angle moyen d'insertion des branches et pourrait correspondre à une caractéristique de l'individu observé. Le déterminisme biologique de cette composante morphologique reste inconnu.

D’un point de vue pratique, 2 mesures simples semblent suffire pour décrire la fourchaison bien qu'elle soit très influencée par les conditions climatiques de l'année, tant du point de vue de l'importance que de la nature et de l'origine des défauts:

- un nombre de pousses secondaires sur branches latérales ou mieux un nombre de pousses secondaires ayant occasionné l'apparition d'un défaut. Ce caractère est facilement mesurable verticille par verticille;

- une note d'angle de branchaison comprise entre 1 et 4 . Cette mesure apporte une information importante sur les causes de la fourchaison autres que le polycyclisme.

\subsection{Variabilité génétique des descripteurs de la fourchaison sur les 48 descendances}

Le nombre de pousses secondaires et l'angle de branchaison, déjà bons descripteurs du phénomène de fourchaison doivent satisfaire à quelques conditions supplémentaires pour être de bons critères de sélection:

- présenter une variabilité génétique suffisante,

- avoir une héritabilité individuelle aussi élevée que possible,

- présenter des corrélations génétiques favorables avec les autres caractères de sélection utilisés. 


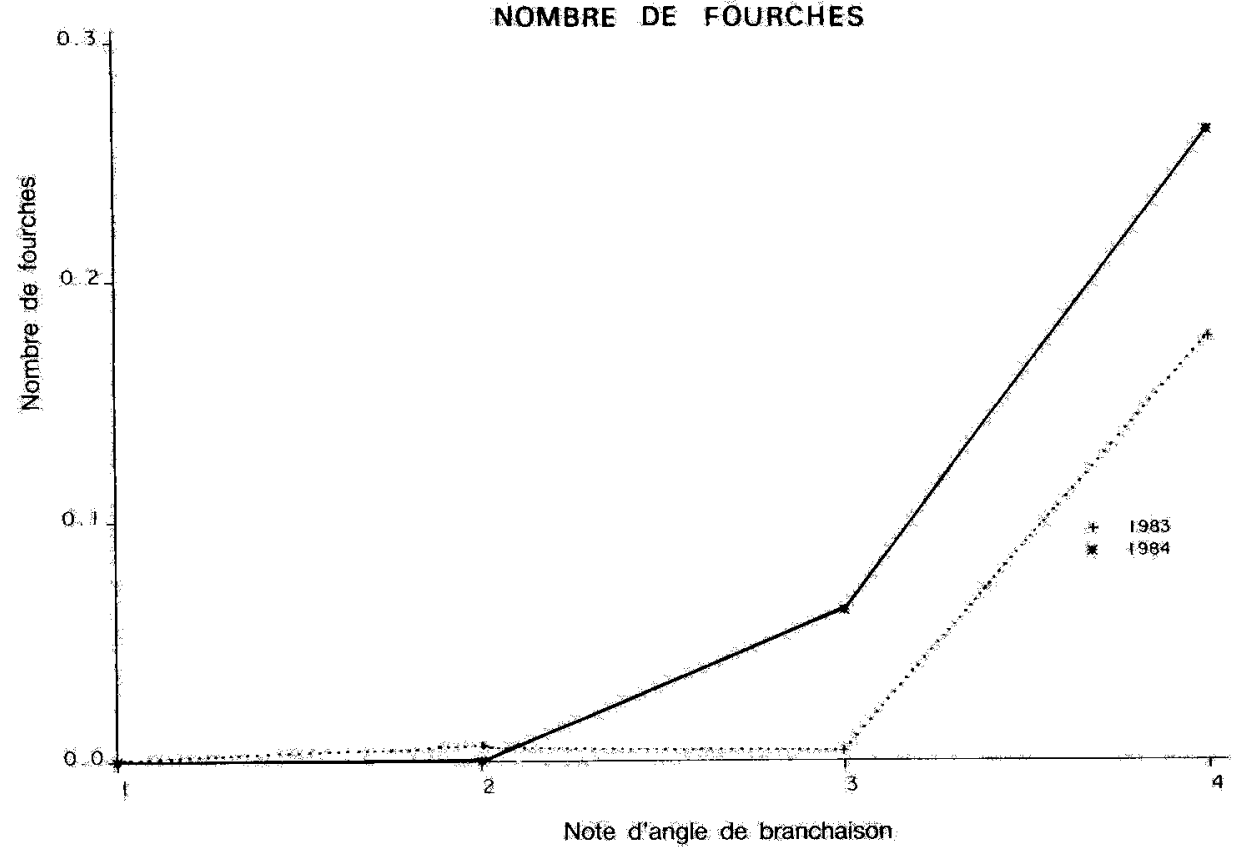

Fig. 5

Valeurs movennes individuelles observées sur 4 descripteurs en fonction de la note d'angle de branchaison atribuée en 1983 et 1984.

Individual means observed for the different values of branch angle score assigned in 1983 and in 1984.

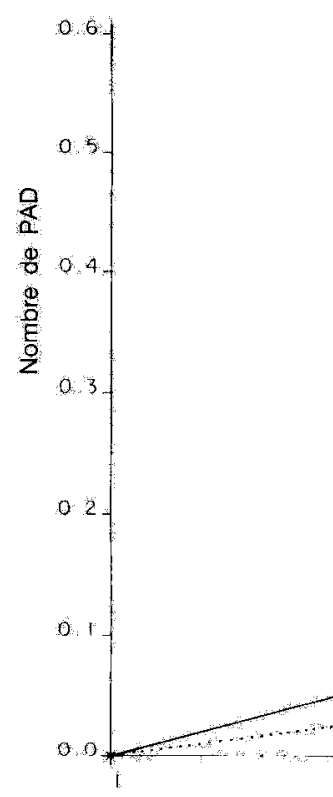

NB. DE POUSSES SECONDAIRES

A $L$ ORIGINE DE DEFAUTS 

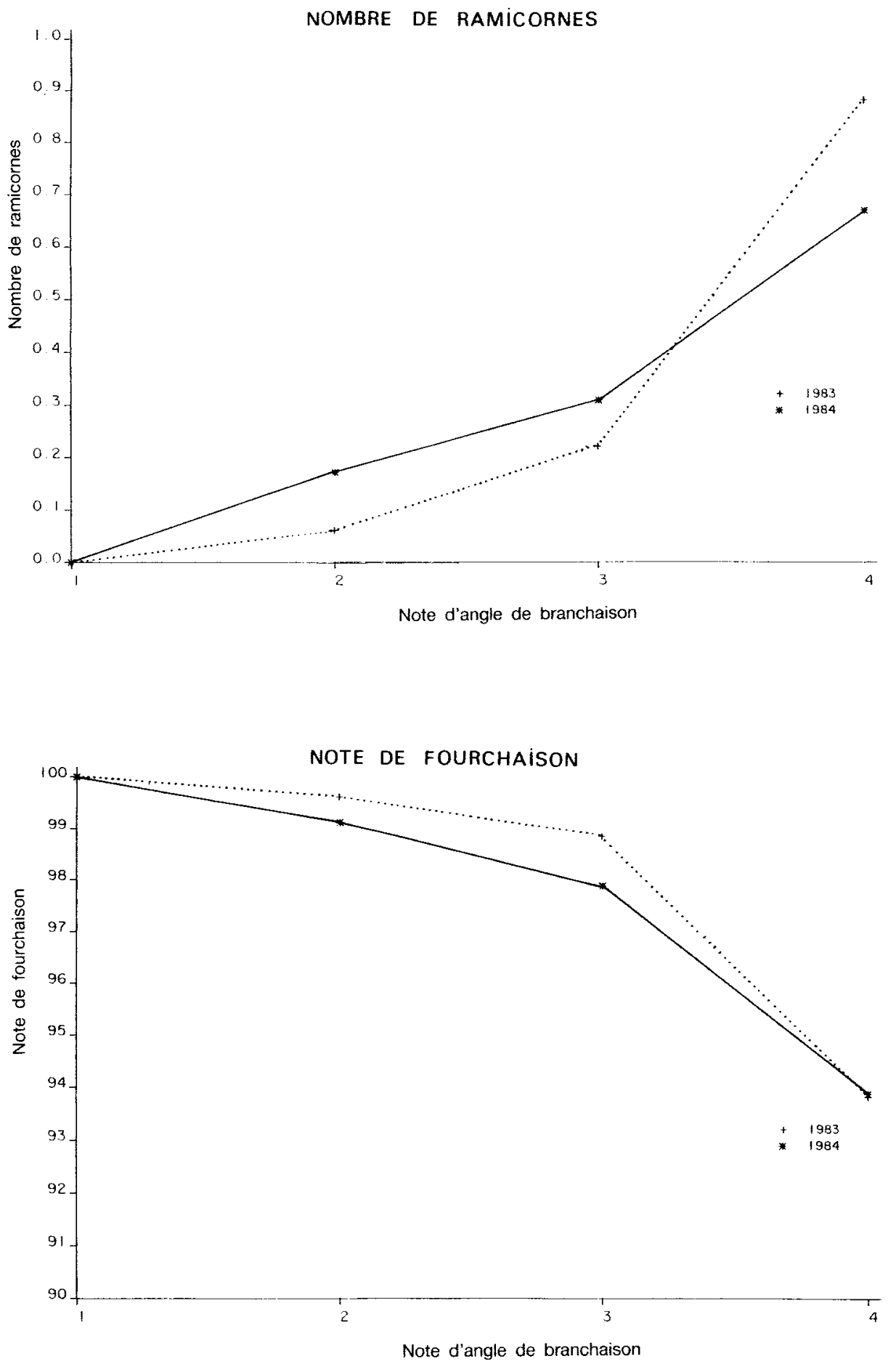


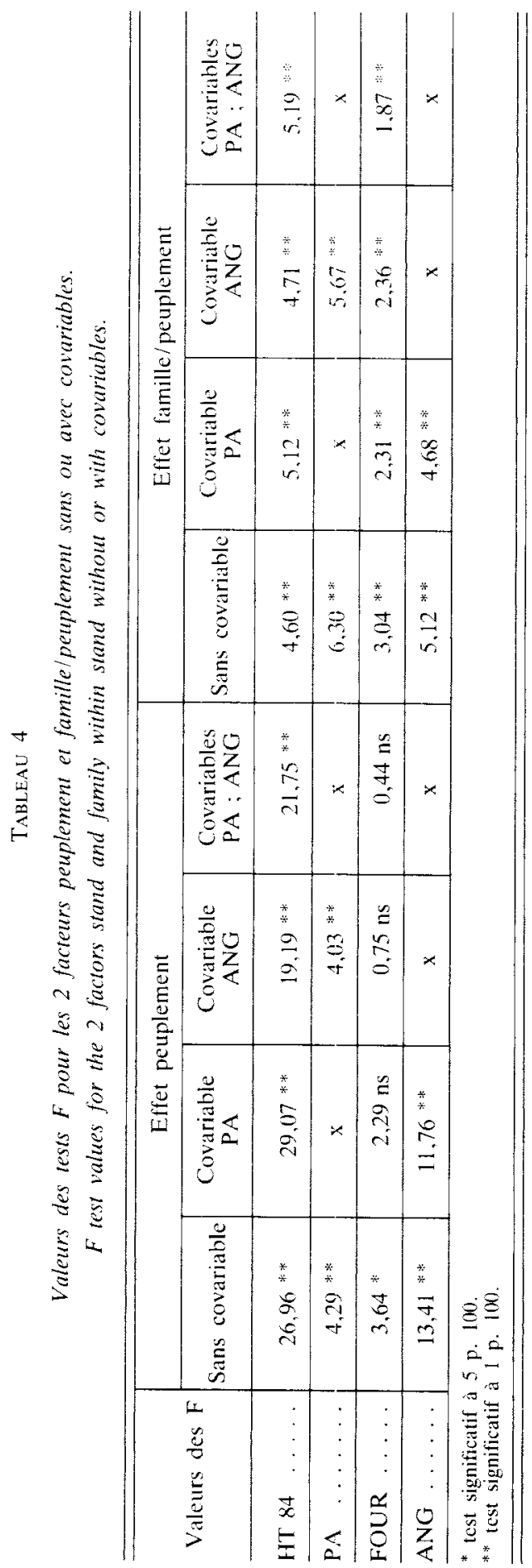


Dans ce dispositif, la vigueur est appréciée par la hauteur totale observée en 1984 , c'est-à-dire à l'âge de 8 ans depuis la graine. Polycyclisme, angle de branchaison et note de fourchaison sont cumulés sur les 2 années 1983 et 1984.

$$
\begin{aligned}
& \mathrm{PA}=\text { PA83 }+ \text { PA84 } \\
& \text { ANG }=\frac{1}{2}(\text { A } 83+\text { A } 84) \\
& \text { FOUR }=\text { FOUR } 83+\text { FOUR } 84-100
\end{aligned}
$$

\subsection{Importance des effets peuplement et famille}

Les valeurs des tests $\mathrm{F}$ obtenues par analyse de variance à 2 facteurs avec et sans ajustement aux deux covariables PA et ANG sont regroupées dans le tableau 4.

- Une partie de la variabilité des 2 caractères FOUR et ANG est imputable à la variabilité observée pour le polycyclisme, ceci aux 2 niveaux peuplement et famille.

- Il est à noter que si l'effet peuplement de la note de fourchaison n'est dû qu'au seul effet de ce facteur sur le polycyclisme, il existe un effet peuplement significatif à 1 p. 100 sur la note d'angle de branchaison même après ajustement à la covariable.

- Toute la variabilité de la note de fourchaison observée au niveau famille ne peut s'expliquer par les 2 facteurs polycyclisme et angle de branchaison.

\subsection{Estimation des paramètres génétiques}

Les variances phénotypiques, interfamilles et résiduelles, ainsi que les héritabilités individuelles sont présentées dans le tableau 5.

\section{Tableau 5}

Estimation des écarts-types phénotypiques (op), des coefficients de variation phénotypiques (CVp). des variances interfamilles $\left(\sigma_{f}^{2}\right)$ et résiduelles $\left(\sigma_{w}^{2}\right)$ et des héritabilités individuelles $\left(h^{2}\right)$.

Estimation of phenotypic standard-deviations (op), phenotypic variation coefficients (CVp) between families $\left(\sigma_{i}^{2}\right)$ and residual $\left(\sigma_{n}^{2}\right)$ variances and individual heritabilities $\left(h^{2}\right)$.

\begin{tabular}{c|c|c|c|c|c}
\hline \hline Caractère & $\sigma \mathrm{p}$ & $\mathrm{CV}_{\mathrm{p}} \mathrm{p} .100$ & $\sigma_{\mathrm{f}}^{2}$ & $\sigma_{\mathrm{w}}^{2}$ & $\mathrm{~h}^{2}$ \\
\hline HT $84 \ldots \ldots$ & 43,72 & 22,5 & 128,93 & 1718,6 & 0,28 \\
\hline PA $\ldots \ldots$ & 2,04 & 151,1 & 0,454 & 4,11 & 0,40 \\
\hline FOUR . . & 7,76 & 8,3 & 2,60 & 61,05 & 0,16 \\
\hline ANG . . . & 0,542 & 20,2 & 0,024 & 0,294 & 0,30 \\
\hline \hline
\end{tabular}

Les valeurs relatives aux 2 caractères de vigueur (HT 84 et PA) sont en accord avec celles obtenues dans les 2 premières tranches du verger à graines du Lot, à 5 et 6 ans (Roman-Amat, 1984).

$$
\begin{gathered}
\text { Tranche } 201 \\
\mathrm{~h}^{2}{ }_{\text {HT } 80}=0,38 \\
\mathrm{~h}^{2} \text { PO } 78.80=0,49
\end{gathered}
$$

Tranche 202

$$
\begin{gathered}
\mathrm{h}_{\text {HT82 }}^{2}=0,35 \\
\mathrm{~h}_{\text {PO 79.81 }}^{2}=0,49
\end{gathered}
$$


Hauteur totale et polycyclisme sont non seulement héritables mais présentent en outre une variabilité phénotypique intéressante pour le sélectionneur.

L'estimation de l'héritabilité de l'angle de branchaison chez le Pin laricio de Corse est proche de celle obtenue à 10 ans sur un test de descendances de Pin laricio de Calabre (Arbez \& Millier, 1972, cf. 2.33.). Dans la population étudiée ici, les coefficients de variation phénotypiques de la note d'angle de branchaison et de la hauteur totale ont le même ordre de grandeur.

L'examen des paramètres calculés pour la note de fourchaison souligne l'intérêt d'une décomposition du phénomène observé : les 2 meilleurs descripteurs de la fourchaison (PA et $\mathrm{ANG}$ ) présentent une héritabilité et une variabilité phénotypique plus élevées que la note de fourchaison elle-même.

Le tableau 6 regroupe les estimations de corrélations génétiques et résiduelles entre les 4 caractères cibles de la sélection.

\section{TABleaU 6}

Corrélations génétiques $\left(r_{1}\right)$ et résiduelles $\left(r_{n}\right)$ calculées sur les 48 descendances maternelles (corrélations génétiques au-dessus de la diagonale).

Genetic correlations $\left(r_{.1}\right)$ and residual correlations $\left(r_{n}\right)$ computed on the 48 open pollinated progenies (Genetic correlations are above the diagonal).

\begin{tabular}{|c|c|c|c|c|c|c|}
\hline$r_{w} \bigcirc r_{A}$ & HT 84 & PA & A 83 & A 84 & ANG & FOUR \\
\hline HT 84 & & 0.382 & -0.023 & 0,126 & 0,060 & -0.124 \\
\hline PA & -0.204 & & 0,180 & 0.260 & 0,230 & -0.666 \\
\hline A $83 \ldots \ldots$ & 0.250 & 0.000 & & 0,898 & 0,969 & $-0,568$ \\
\hline A 84 & 0.185 & 0.026 & 0.410 & & 0,979 & $-0,680$ \\
\hline ANG & 0.257 & -0.016 & 0.828 & 0,851 & & $-0,646$ \\
\hline FOUR $\ldots$ & 0.041 & -0.412 & $-0,299$ & -0.419 & $-0,430$ & \\
\hline
\end{tabular}

La corrélation génétique positive entre hauteur et polycyclisme est significative mais s'expliquerait plutôt par un déterminisme voisin des 2 caractères en conditions favorables, que par une participation réelle des pousses secondaires à l'élongation totale de l'arbre (cf. 2.222.). Cette corrélation semble expliquer une grande partie de la liaison défavorable vigueur-fourchaison (ici HT84-FOUR), la corrélation note d'angle de branchaison-hauteur totale n'étant pas significative. L'estimation de cette dernière chez le Pin laricio de Calabre n'était pas significative non plus (Arbez \& Milluer, 1972).

Les 2 valeurs d'angle de branchaison observées en 1983 et 1984 sont fortement corrélées entre elles, tant au niveau inter, quintra famille. Ce résultat confirme la stabilité intra arbre de ce caractère au cours du temps. 
En termes de gains génétiques attendus par sélection, l'utilisation des 2 prédicteurs polycyclisme et angle de branchaison apparaît particulièrement efficace dans la population d'amélioration étudiée.

Une contre sélection du polycyclisme permet d'espérer, par sélection indirecte, le même gain génétique sur la note de fourchaison que celui attendu par sélection directe soit 2,3 p. 100 pour un taux de sélection de 10 p. 100.

Les 2 caractères hauteur et angle de branchaison étant indépendants, un gain génétique simultané pourra être attendu sur la vigueur et sur le niveau de fourchaison par l'intermédiaire de l'angle de branchaison.

\subsection{Discussion}

Pour un programme d'amélioration où vigueur et fourchaison sont les principaux objectifs, et pour la population étudiée ici, hauteur totale à 8 ans, angle de branchaison et polycyclisme semblent être les 3 meilleurs critères de sélection. La sélection combinée en faveur de la croissance en hauteur êt contre l'apparition de pousses secondaires se heurte à une corrélation génétique défavorable. Deux stratégies sont cependant possibles :

1) Les conditions de milieu dans lesquelles seront plantés les descendants des individus sélectionnés sont en général peu favorables à l'expression du polycyclisme. Une des principales causes de la fourchaison est alors éliminée. Une sélection combinée sur les 2 caractères hauteur totale et angle de branchaison permet d'attendre un gain maximum sur la vigueur, tout en améliorant le niveau de fourchaison.

2) Les conditions de culture envisagées sont plutôt intensives et favorisent l'apparition de pousses secondaires fréquemment à l'origine de défauts de fourchaison. Une telle situation interdit l'obtention du gain génétique maximum sur la vigueur. Toutefois, la construction d'un indice de sélection combinant la hauteur totale, le polycyclisme et l'angle de branchaison permet la réalisation du meilleur compromis: un progrès génétique optimum est attendu tant sur la vigueur que la fourchaison.

Index de sélection utilisé

\begin{tabular}{|c|c|c|c|}
\hline & $\mathrm{E}\left\{\Delta \mathrm{G}_{\mathrm{H}[\mathrm{s} \psi}\right\}$ & $\mathrm{E}\left\{\Delta \mathrm{G}_{\mathrm{FOUR}}\right\}$ & $\mathrm{E}\left\{\Delta \mathrm{G}_{\mathrm{ANG}}\right\}$ \\
\hline $\begin{array}{l}\text { 1) } \mathrm{I}=\hat{\mathrm{G}}_{\mathrm{HIXA}}+1,475 \hat{\mathrm{G}}_{\mathrm{FOUR}} \ldots \ldots \ldots \\
\text { 2) } \mathrm{I}=15 \hat{\mathrm{G}}_{1 \mathrm{ITY} A}-4,7 \hat{\mathrm{G}}_{\mathrm{PA}}-2,0 \hat{\mathrm{G}}_{\mathrm{ANG}} \ldots \ldots\end{array}$ & $\begin{array}{l}12,4 \text { p. } 100 \\
13,8 \text { p. } 100\end{array}$ & $\begin{array}{r}0 \text { p. } 100 \\
+0.5 \text { p. } 100\end{array}$ & $\begin{array}{l}-0,8 \text { p. } 100 \\
-1,62 \text { p. } 100\end{array}$ \\
\hline
\end{tabular}

\section{Conclusion}

Cette étude confirme le rôle essentiel joué par le polycyclisme dans l'apparition des fourches et ramicomes, observées chez le Pin laricio de Corse dans les conditions environnementales de la forêt d'Orléans. La liaison entre le nombre de pousses secondaires et l'ampleur des défauts varie cependant avec le type de polycyclisme observé, très sensible à l'effet année. 
L'angle de branchaison, mesure plus stable dans le temps, permet d'apprécier un angle moyen d'insertion des branches au niveau d'un ou plusieurs verticilles, tout en tenant compte de l'importance de la limite inféricure de $30^{\circ}$, à l'origine de la définition des 2 types de défauts.

L'utilisation de ces 2 composantes dans un programme d'amélioration génétique permet de réduire l'antagonisme existant entre gain sur la vigueur et gain sur la fourchaison. Aussi, l'espérance de gain génétique attendue sur la croissance en hauteur s'en trouve-t-elle sensiblement accrue.

Reçu le 29 décembre 1986.

Accepté le 15 avril 1987.

\begin{abstract}
Summary
Components of forking observed on corsican pine: interest in a breeding program (Pinus nigra Arn. ssp. laricio l'ar. corsicana Loud)
\end{abstract}

Two types of forking defects, fork and ramicorne can seriously affect stem quality of Corsican Pine. The use of a forking score between 0 and 100 as selection criterion seemed not to be very efficient for the breeding population studied (tabl. 1). The purpose of this work is to find through factors explaining forking, one or several good predictors ensuring maximum genetic gain.

Measurements were made in fall 1985 on a 9 years old open pollinated progeny test located in Orléans forest. The importance of three already known explicative factors of forking defects was studied for the years 1983 and 1984 on a sample of 443 forked trees.

- Insect attacks (Rhyacionia buoliana) and rust damages.

- Polycyclism, through the total number of litteral lammas shoots (PA) and lateral lammas shoots inducing defects (PAD),

- Average branch angle with a special notation scale (fig. 1).

Genetic parameters (family and residual variances, individual heritabilitics, genetic and residual correlations) were computed on 48 half-sib families after an analysis of variance with a hierarchical model (tabl. 2).

Insect attacks and rust damages can't explain forking defects either in 1983 or in 1984.

Although it is affected by climatic conditions, polycyclism seems to be the main explanatory forks of (presence of forks and ramicornes) in 1983 and 1984. Meanwhile, defects are rather due to a secondary elongation of some lateral buds, than to real lammas shoots (fig. 3).

The branch angle score used, discriminates well the most forking trees (fig. 5) and can take into account factors unducing forks independent of polycyclism. This measure is more stable from one year to another and better describes a characteristic of each tree.

In the studied breeding population, polycyclism and branch angle explain a great proportion of the observed variability for the forking score (tabl. 4). Meanwhile, because of their high values of between families variances and individual heritabilities, these 2 traits seem to be more interesting for the breeder than the forking score (tabl. 5). It results, from the non significative genetic correlation between total height and branch angle that mark a simultaneous genetic gain can be obtained on both traits (tab). 6). This progress is limited by the genetic positive unfavorable correlation between polycyclism and total height and the best selection compromise need the use of selection index combining the 3 characters.

Key words : Pinus nigra ssp. laricio v'ar corsicana, jorking, polycyclism, branch angle, heritability. genetic variance, genetic correlation, genetic gain. 


\section{Références bibliographiques}

Arbez M., Millier C., 1972. Variabilité, héritabilité et corrélations entre caractères chez de jeunes Pins laricio de Calabre (Pinus nigra Arn. ssp. laricio, var. calabrica). Conséquences et problèmes des indices de sélection. Proceedings of the I.U.F.R.O. Genetics. S.A.B.R.A.O. Joint Symposium Tokyo.

CARvfil L.K., 1956. Summer shoots cause permanent damage to Red pine. Journal of Forestry, 54 (4), p. 271.

Crisan P.A., 1977. Déterminisme génétique de la forme du Pin maritime. Corrélations avec la vigueur et le rythme de croissance. Rapport de stage de $3^{c}$ année E.N.I.T.E.F., INRA L.A.A.F. doc. interne.

Ehrenberci C.E., 1963. Genetic variation in Progeny tests of Scots Pinc (Pinus sylvestris L.). Studia forestalia suecica, $\mathrm{n}^{\text {"1 }} 10$.

FAlconer D.S., 1974. Introduction à la génétique quantitative. Masson, Editeur, Paris.

Kullback S., 1968. Information theory and statistics. Dover Publications, Inc. New York.

JARRET P., 1978. Variabilité génétique des caractères de forme (tronc et branchaison) et de quelques caractères auxométriques et phénologiques chez le Douglas. Rapport de stage de $3^{c}$ année E.N.I.T.E.F., INRA S.A.A.F. doc. interne.

Rabat P., 1985. Contribution à l'étude de la croissance et des anomalies de branchaison chez le Pin laricio en plantation. Rapport de stage de $3^{\circ}$ année E.N.I.T.E.F.

Roman-Amat B., 1984. Contribution à l'exploration et à la vaiorisation de la variabilité intraspécifique et individuelle du Pin laricio de Corse, Pinus nigra arn. ssp. laricio, var corsicana Loud. Thèse de Docteur-Ingénieur soutenue devant l'Université de Paris-Sud, Centre d'Orsay.

RudolPH T.D., 1964. Lammas growth and prolepsis in Jack pine in the Lake States. Forest Science $n^{\text {" }} 6$.

West R.F., Ledig F.T., 1964. Lammas shoot formation in Scoteh pine. 11th Northeastern Forest Tree Improvement conference proceedings, pp. 21-30. 